Letrônica, Porto Alegre, v. 7, n. 1, p. 250-277, jan./jun., 2014

\title{
RECURSIVIDADE NA SINTAXE DA LÍNGUA E NA ARITMÉTICA: INTERDEPENDÊNCIA OU INDEPENDÊNCIA ENTRE DOMÍNIOS? UM ESTUDO EXPERIMENTAL ${ }^{1}$
}

\author{
RECURSION IN LANGUAGE SYNTAX AND ARITHMETICS: \\ INTERDEPENDENCE OR INDEPENDENCE BETWEEN DOMAINS? \\ AN EXPERIMENTAL STUDY
}

\author{
Mercedes Marcilese* \\ Letícia Maria Sicuro Corrêa** \\ Marina Rosa Ana Augusto***
}

\begin{abstract}
Resumo: A capacidade de computar estruturas hierárquicas recursivas, tais como as encontradas nas gramáticas naturais, tem sido apontada como um dos principais aspectos que separa os humanos de outros primatas (HAUSER; CHOMSKY; FITCH, 2002). Esse tipo de estrutura, no entanto, não parece ser exclusivo do domínio das línguas naturais humanas. Em termos estritamente estruturais, um paralelo pode ser traçado entre determinadas sentenças linguísticas e expressões numéricas. Especificamente, em ambos os casos, podem ocorrer estruturas envolvendo center-embedding. No presente artigo, a noção de recursividade linguística - tal e como concebida no âmbito da Teoria Gerativa - é examinada e são reportados os resultados de um experimento concebido com o objetivo de explorar um possível efeito de priming estrutural no processamento de estruturas recursivas associadas a domínios cognitivos diferentes, quais sejam, a cognição linguística e numérica. Os dados obtidos não sustentam a ideia de que o processamento de estruturas semelhantes associadas a domínios diferentes seja afetado por um efeito de priming de natureza estrutural. Esses resultados são discutidos à luz do
\end{abstract}

\footnotetext{
${ }^{1}$ A pesquisa aqui relatada foi desenvolvida no âmbito da tese de doutorado na primeira autora, então bolsista CNPq, sob orientação e coorientação das segunda e terceira autoras, respectivamente. A investigação é parte do Projeto FAPERJ Edital Humanidades - (Processo E-26/ 112.273/2008-2010), desenvolvido no âmbito das atividades do GPPAL (Grupo de Pesquisa em Processamento Linguístico e Aquisição da Linguagem/CNPq), no LAPAL (Laboratório de Psicolinguística e Aquisição da Linguagem) da PUC-Rio. A redação do artigo foi feita na vigência do projeto CNPq-PQ 308874/2011-0.

* Doutora pela Pontifícia Universidade Católica do Rio de Janeiro (PUC-Rio). Professora Adjunta da Universidade Federal de Juiz de Fora, atuando na Graduação, na Pós-Graduação em Linguística e no Núcleo de Estudos em Aquisição da Linguagem e Psicolinguística (NEALP), e participante do GPPAL/LAPAL.

${ }^{*}$ PhD pela University of London, Professora Associada Sênior do Departamento de Letras da PUC-Rio, onde atua na Graduação e na Pós-Graduação, na área de Psicolinguística, e como coordenadora do LAPAL.

*** Doutora em Linguística pela Universidade Estadual de Campinas (UNICAMP). Professora Adjunta da Universidade do Estado do Rio de Janeiro (UERJ) onde atua na Graduação e na Pós-Graduação, e membro do GPPAL/LAPAL.
} 
conceito de interface em um modelo de computação linguística on-line e de evidências da neurociência da linguagem.

Palavras-chave: Recursividade; Língua; Cognição Numérica; Priming Estrutural.

Abstract: The ability to compute hierarchical recursive structures, such as those found in natural grammars, has been identified as a key feature for distinguishing humans from other primates (HAUSER; CHOMSKY; FITCH, 2002). This kind of structure, however, does not seem to be exclusive to the domain of natural language. In strictly structural terms, a parallel can be drawn between linguistic sentences and numerical expressions. Specifically, in both cases there may be structures with center-embedding. This paper aims to address the notion of linguistic recursion, within the framework of Generative Theory, and to report experimental results. A self-paced reading experiment was designed to explore a priming effect in the processing of recursive structures associated with different cognitive domains, namely, language and numerical cognition. The data do not support the idea that the processing of similar structures is affected by cross-domain syntactic priming. The results are discussed in the light of a concept of an interface between an on-line model of linguistic computation and of current evidence from the field of neuroscience.

Keywords: Recursion; Language; Numerical Cognition; Syntactic Priming.

\section{Introdução}

A natureza recursiva da sintaxe tem sido destacada, no âmbito da Teoria Gerativa desde os anos 1950, como uma das propriedades fundamentais das línguas naturais. As analogias entre os sistemas numérico e linguístico baseadas nessa propriedade não são raras na literatura (CHOMSKY, 1998; 2007). Crucialmente, línguas humanas e sistemas numéricos compartilham a propriedade da infinitude discreta (CHOMSKY, 1998; HAUSER; CHOMSKY; FITCH, 2002; CORVER et al., 2007). Em outras palavras, assim como séries de números podem ser indefinidamente continuadas - na medida em que sempre é possível adicionar "mais um"- também é possível criar novas estruturas sintáticas pela adição de material linguístico a uma estrutura dada (HAUSER; CHOMSKY; FITCH, 2002). Nesses termos, a propriedade da infinitude discreta permite explicar o fato de que não há limites previamente estabelecidos para o número de elementos que uma sentença da língua ou uma sequência numérica possa conter.

Cabe destacar que, apesar de ser um termo de uso comum no âmbito do gerativismo, o conceito de recursividade, aplicado tanto ao domínio da língua quanto a outros campos, não tem recebido uma definição clara e unívoca. Observa-se ainda que, até relativamente pouco tempo atrás, não havia na literatura uma preocupação por esclarecer os pontos 
obscuros associados à noção. Essa situação tem começado a mudar com a publicação recente de trabalhos que discutem os alcances do conceito, tanto no seio da Teoria Linguística quanto no que diz respeito à sua aplicação no contexto das Ciências Cognitivas de um modo mais geral (TOMALIN, 2007; LOBINA; GARCÍA-ALBEA, 2009; ARSENIJEVIC; HINZEN, 2010; MARCILESE, 2011).

A habilidade de processar estruturas hierárquicas tais como as encontradas nas gramáticas naturais pode ser vista como um dos aspectos que separam os humanos de outros primatas (HAUSER; CHOMSKY; FITCH, 2002). As línguas humanas apresentam relações hierárquicas e de longa distância e, crucialmente, admitem o encaixamento recursivo de sintagmas dentro de outros sintagmas (HAUSER; CHOMSKY; FITCH, 2002). Resultados experimentais sugerem que humanos são capazes de aprender tanto gramáticas artificiais de estado finito (Finite State Grammar) quanto gramáticas de estrutura frasal (Phrase Structure Grammar - associadas a estruturas hierárquicas), enquanto primatas não humanos seriam capazes de lidar apenas com o primeiro tipo de gramáticas (FITCH; HAUSER, 2004).

Friederici et al. (2006a) trazem resultados compatíveis com a ideia de que a área de Broca - junto com uma região cerebral filogeneticamente mais antiga, relacionada ao processamento de gramáticas de estado finito - atuaria na computação de gramáticas de estrutura frasal. Esses resultados sugerem que o processamento de estruturas hierárquicas, tais como as presentes nas gramáticas naturais, está associado a áreas específicas do cérebro. Cabe observar, nessa direção, que a hipótese de a recursividade ser uma propriedade exclusiva da faculdade de linguagem em sentido restrito e, como tal, ausente de sistemas de comunicação animal (HAUSER; CHOMSKY; FITCH, 2002), não imediatamente implica que não se encontrem operações recursivas em outros domínios da cognição (como cognição espacial, cognição numérica, etc.) (MARCILESE, 2011).

0 conceito de recursividade na Teoria Linguística, como propriedade fundamental ou inerente às línguas humanas, que, recentemente, deu origem a polêmicas (cf. a discussão levantada em Everett, 2005, 2007 e Nevins, Pesetsky e Rodrigues, 2007, dentre outros), se tem tornado foco de crescente interesse, tanto no que concerne à sua relação com diferentes domínios da cognição, como em pesquisas vinculadas ao estudo de línguas ainda 
pouco conhecidas. ${ }^{2} \mathrm{O}$ presente trabalho se propõe a examinar alguns aspectos relativos ao tratamento dado ao conceito de recursividade na linguística gerativista e avaliar - de forma ainda bastante exploratória, por meio de metodologia experimental - a possibilidade de a recursividade - tal e como caracterizada quando associada ao domínio da língua - poder ser considerada uma propriedade compartilhada por outros domínios da cognição, sendo a cognição numérica o foco do nosso interesse atual.

A possibilidade de compartilhamento de estruturas recursivas em diferentes domínios tem sido recentemente explorada (PESETSKY; KATZ, submetido; BARTLET; KAZAKOV, 2004; KAZAKOV; BARTLET, 2005; ARSENIJEVIC, 2008; dentre outros). Enquanto há argumentos que sustentam a hipótese de que operações comuns atuam sobre bases de dados distintas (PESETSKY; KATZ, submetido; SCHNEIDER et al., 2011), evidências obtidas em estudos a base de imagem cerebral são interpretadas como compatíveis com a hipótese de haver recursos neuronais distintos para processos semelhantes em diferentes domínios (FRIEDRICH; FRIEDERICI, 2009), no que diz respeito à computação de estruturas hierarquicamente organizadas. No entanto, independentemente dos circuitos neuronais que implementem procedimentos recursivos em diferentes domínios, sua realização pode depender de recursos de memória compartilhados. 0 trabalho conduzido por Fedorenko, Gibson e Rohde (2007), por exemplo, sugere que a memória verbal de trabalho estaria envolvida tanto no processamento de sentenças da língua, quanto de expressões numéricas, sugerindo certo grau de compartilhamento de recursos entre ambos os domínios.

0 presente artigo se insere nessa discussão ao verificar em que medida tal tipo de compartilhamento poderia provocar interferência entre domínios. Este está organizado da seguinte forma: em primeiro lugar, os sentidos atribuídos ao termo recursividade no âmbito da Linguística Gerativa são considerados, com vista a uma definição que possibilite a comparação de processos recursivos entre domínios cognitivos. Em seguida, é avaliada a possibilidade de haver um compartilhamento de recursos entre sistemas cognitivos diferentes, envolvendo a recursividade como propriedade comum. Na seção 3, são reportados os resultados de um experimento conduzido com o objetivo de explorar uma

${ }^{2}$ Cf. Trabalhos apresentados no evento Recursion in Brazilian Languages and Beyond, realizado em 2013 na UFRJ (cf. http://www.letras.ufri.br/poslinguistica/recursion/\# event-program). 
possível relação mais direta entre o processamento de estruturas recursivas nos domínios linguístico e numérico. Esses resultados são discutidos à luz do conceito de informação das interfaces entre a língua e sistemas de desempenho, na computação on-line e diante de resultados da neurociência cognitiva da linguagem, ao que se seguem as considerações finais.

\section{Recursividade na Teoria Linguística}

As definições de recursividade encontradas na Teoria Linguística são muitas vezes opacas, sendo que esse não constitui um problema exclusivo da Linguística já que também na Ciência da Computação - campo do qual a noção foi herdada - as definições não apresentam um fio condutor único (PARKER, 2006a, 2006b). Também na Matemática, âmbito no qual o termo tem sua origem, registra-se uma situação similar (SOARE, 1996). Recursividade apresenta-se assim como um termo potencialmente problemático (PARKER, 2006a, 2006b; LOBINA; GARCÍA-ALBEA, 2009; dentre outros).

Embora a Matemática e a Ciência da Computação compartilhem uma caracterização mais geral de recursividade, é possível estabelecer algumas distinções em cada caso. Primeiramente, a noção de recursividade surge no campo da Matemática associada à ideia de um método para definir funções (e outros objetos ou relações). A recursividade, nesse contexto, é caracterizada como uma propriedade de certos objetos, relações ou mecanismos (Ex. "conjuntos recursivos", "algoritmos recursivos", "funções recursivas", "problemas com soluções recursivas", "relações recursivas", "definições recursivas", etc.). O conceito aparece, nesse contexto, definido de forma bastante geral:

A method of defining functions studied in the theory of algorithms and other branches of mathematical logic. This method has been used for a long time in arithmetic to define sequences of numbers (progressions, Fibonacci numbers, etc.). Recursion plays an important role in computational mathematics (recursive methods). Finally, in set theory transfinite recursion is often used. For a long time the term 'recursion' was used by mathematicians without being accurately defined (VINOGRADOV, 1992, p. 15).

No âmbito da Ciência da Computação, recursividade constitui uma ferramenta ou uma técnica de programação. Parker (2006a, 2006b) chama atenção para o fato de que na 
Computação as definições de recursividade carecem de um fio condutor único e estabelece uma distinção entre as definições encontradas. Enquanto algumas definições enfatizam o fato de haver a repetição de um dado processo, como (1) e (2), outras como (3) consideram a auto-referência como a característica mais marcante (i.e. o fato de que um objeto seja definido em termos de si mesmo). Por último, algumas definições centram-se no fato de que determinados processos ou procedimentos podem se invocar a si mesmos - ou, colocado em termos computacionais, um algoritmo ou método pode se "chamar a si mesmo" (4).

(1) Recursion and iteration are two equivalent ways in programming for repeatedly performing a specific task (LOEPER et al. (1996, p.153) apud PARKER, 2006a).

(2) Recursion and iteration both result in the controlled repeated execution of a body of code (ARNOW; WEISS, 1998, p. 494).

(3) Recursion allows something to be defined in terms of smaller instances of itself (LOUDON, 1999).

(4) Recursion is the ability of a subroutine or procedure to call itself (HAREL, 1993, p. 31).

A noção de recursividade adotada na Linguística é herança da Ciência da Computação e tem ocupado historicamente um lugar de destaque na Teoria Gerativa como meio de expressar formalmente, em uma gramática gerativa, a propriedade da infinitude discreta (i.e. a possibilidade de que um número potencialmente infinito de sentenças seja gerado a partir de um conjunto discreto de elementos, assim como também a possibilidade de uma estrutura sintática poder abrigar elementos que se sucedem recursivamente). No âmbito da Linguística, o termo também aparece comumente associado à noção de produtividade ou criatividade linguística, embora apenas o primeiro termo esteja estritamente relacionado à noção de recursividade.

A Teoria Linguística parece ter, por um lado, adotado inicialmente o conceito como uma ferramenta formal para reduzir a complexidade da gramática (ĆAVAR; ĆAVAR, 2007) e, por outro, como um meio de enfatizar a capacidade produtiva das línguas humanas: "If a grammar has no recursive steps [...] it will be prohibitively complex [...]. If it does have recursive devices, it will produce infinitely many sentences" (CHOMSKY, 1956, p. 116).

Desde Chomsky (1975 [1955]; 1957), a necessidade de uma gramática gerativa incorporar recursos formais que viabilizem a expressão de seu caráter recursivo é enfatizada. Em Chomsky (1975 [1955]), o componente sintagmático da gramática (Phrase 
Structure Component) é apresentado em termos de uma sequência de regras do tipo $\mathrm{X} \rightarrow \mathrm{Y}$ aplicadas iterativamente, como meio de expressar o caráter recursivo das línguas (cf. TOMALIN, 2007). Em Chomsky (1957), transformações generalizadas apresentam-se como um possível recurso para o encaixamento de orações. Já em (1965), a recursividade no procedimento gerativo é diretamente expressa em regras de reescritura (i.e. do tipo $\mathrm{X} \rightarrow \mathrm{Y}$ ), nas quais um elemento inicialmente apresentado à esquerda do símbolo de reescritura " $\rightarrow$ " pode reaparecer, em outra regra ou na mesma, à direita deste (Ex.: S $\rightarrow$ NP VP; NP $\rightarrow$ D N S...), ficando, portanto, o potencial de infinitude discreta das sentenças da língua formalmente expresso no nível representacional anterior à aplicação de regras transformacionais (isto é, as regras de reescritura são codificadas no nível da Estrutura Profunda). Com a eliminação desse nível de representação, na fase minimalista do gerativismo chomskyano, soluções que retomam a ideia de aplicação iterativa de regras (no caso Merge) ou uma releitura das transformações generalizadas (cf. Lighfoot em Chomsky, 2002 [1957]) são buscadas na simplificação da formalização da gramática.

A principal diferença com relação ao modo como recursividade se apresenta em um dado modelo de gramática diz respeito basicamente ao formalismo utilizado. Em outras palavras, quando se diz atualmente que os traços de um núcleo são projetados quando este se combina (Merge) com outro elemento, tem-se uma expressão equivalente à reintrodução de um símbolo numa regra de reescritura. Cumpre salientar que a direcionalidade da derivação foi afetada com essa mudança, em relação aos primeiros modelos derivacionais propostos, passando de top-down a bottom-up (direcionalidade esta que incorpora o sentido ascendente das regras sintagmáticas que expressam a projeção dos traços de categorias do léxico a níveis hierarquicamente mais altos (X-barra) nas versões representacionais do modelo de gramática concebidas nos anos 1980).

A noção de recursividade apresentada no contexto da TL veicula os seguintes aspectos: (i) a propriedade de infinitude discreta das sentenças geradas ou do conjunto de sentenças gerado pela gramática; (ii) a representação formal da estrutura frasal das gramáticas cujas sentenças possuem a propriedade explicitada em (iii); (iii) a possibilidade de encaixamento de constituintes do mesmo tipo; (iv) a aplicação iterativa de um regra ou operação. Pode-se encontrar, por exemplo, uma definição semelhante à fornecida na Ciência 
da Computação, contextualizada em termos de estrutura frasal, como a que se encontra em Pinker e Jackendoff (2005, p. 3): "Recursion refers to a procedure that calls itself, or to a constituent that contains a constituent of the same kind".

De forma análoga, em Chomsky (1965), o procedimento que chama a si mesmo fica explícito no formalismo em (ii), do qual uma possível realização é ilustrada em (i). Outras caracterizações de recursividade agregam todos os aspectos anteriormente mencionados (cf. PARISI, 1981).

From the point of view of linguistic theory, the concept of recursion could be defined in both a narrow sense and in a broader sense. In the narrow sense, recursion implies subordination.

(i) I think/hope/promise that John will come tomorrow $\mathrm{S} \rightarrow[\ldots \mathrm{VS}]$

In a broad sense, recursion as a computational system could be defined in logical terms as follows:

(ii) $\mathrm{X} \rightarrow[\ldots . . . . .$.$] (CHOMSKY, 1965, p. 211).$

La ricorsività sintattica significa che una determinata categoria compare come elemento all'interno di un sintagma appartenente a questa stessa categoria. L'esempio più tipico di questo fenomeno sono le frasi che compaiono all'interno di altre frasi. [...] La ricorsività sintattica significa che le stesse regole sintattiche possono venir riapplicate ciclicamente all'interno di una stessa frase. In questo modo si riesce a chiarire come la sintassi del linguaggio umano sia uno dei principali fattori che spiegano il carattere aperto di questo particolare sistema di comunicazione, cioè la sua capacità di generare frasi sempre nuove (PARISI, 1981, p. 25-26).

É importante lembrar que é com base na propriedade da infinitude discreta que mais frequentemente é estabelecido um paralelo entre língua e o sistema dos números naturais:

Human language is based on an elementary property that also seems to be biologically isolated: the property of discrete infinity, which is exhibited in its purest form by the system of natural numbers $1,2,3 \ldots$ Children do not learn this property of the number system. Unless the mind already possesses the basic principles, no amount of evidence could provide them, and they are completely beyond the intellectual range of other organisms. (CHOMSKY, 2000, p. 3-4).

Embora as noções mencionadas aparentemente estabeleçam uma relação de quase sinonímia no seio da teoria, sendo intercambiáveis em determinados contextos, algumas distinções relevantes podem ser estabelecidas. A primeira diz respeito à diferença entre 
recursividade e iteração. A iteração aparece, sob um ponto de vista mais amplo, como o tipo mais simples de recursividade. No domínio da língua, contudo, uma distinção entre ambos os processos pode ser estabelecida com base na diferença entre encaixamento e repetição. Isto é, enquanto a iteração implica a repetição de uma ação ou de um objeto um número arbitrário de vezes, a recursividade envolve o encaixamento de uma ação ou objeto em outra instância de si mesmo. Cabe ainda salientar que a construção da estrutura frasal não precisa necessariamente estar vinculada a regras recursivas (LOBINA; GARCÍA-ALBEA, 2009). Especificamente, a estrutura frasal se refere à relação hierárquica dos constituintes na sentença, ao passo que uma estrutura pode ser hierarquicamente organizada sem por isso ser, necessariamente, recursiva. Na perspectiva de Parker (2006b), a diferença crucial aqui radica no fato de que a hierarquia implica o encaixamento de constituintes em outros constituintes, enquanto a recursividade envolve o encaixamento de constituintes idênticos (Ex. um CP dentro de um CP - [сP Maria acha [ср que vai chover]] -, um PP dentro de um DP dentro de um PP - [DP A menina [Pp $d(\mathrm{e})$ [DP o chapéu [pP de palha]]], etc.).

Adicionalmente, pode ser feita uma distinção com relação às características específicas do encaixamento numa dada estrutura recursiva. Essa distinção é estabelecida em termos de recursividade por ramificação à esquerda/direita (tail) ou no centro (centerembedding). Nestas, o encaixamento duplo dá origem às chamadas "nested structures". A construção possessiva em (5) e a oração relativa em (6) seriam exemplos do primeiro tipo. Já a construção com o duplo encaixamento de uma oração relativa no centro em (7) ilustraria a nested recursion.

(5) 0 irmão da vizinha da amiga da Ana trabalha na novela das sete.

(6) Este é o rapaz que comprou o anel que a Maria tinha visto na vitrine da loja.

(7) 0 rato que o gato que o cachorro mordeu perseguiu escapou ${ }^{3}$.

Em síntese, pode-se dizer que não há, de fato, incompatibilidade entre as diferentes definições propostas, ainda que não haja total equivalência entre os diferentes conceitos com que o de recursividade é identificado. A diversidade superficial radica basicamente no aspecto destacado em cada caracterização: ora a recursividade é colocada como um

\footnotetext{
${ }^{3}$ Apesar de gramatical, o grau de aceitabilidade de frases como (7) é alvo de controvérsia na literatura. Para uma discussão desse aspecto, remetemos aos textos de Karlsson (2010) e Ferreira da Silva (2014). Em termos de processamento, a prosódia parece ter um papel fundamental na compreensão desse tipo de estruturas.
} 
fenômeno linguístico - vinculada assim a propriedades específicas das línguas humanas ora como mecanismo ou recurso computacional, abrangendo também outros possíveis domínios.

A questão de a recursividade ser ou não uma propriedade presente em outros domínios cognitivos, e ainda, a possibilidade de ser compartilhada por outras espécies tem sido, como mencionado anteriormente, discutida na literatura recente (HAUSER; CHOMSKY; FITCH, 2002, 2005). Vários espaços nos quais essa propriedade poderia estar presente têm sido apontados (CORBALLIS, 2007), quais sejam: a cognição numérica, o domínio da visão, a música, a cognição espacial e a teoria da mente. A capacidade de lidar com o conceito de número natural e com operações numéricas, vinculada à cognição numérica é um dos candidatos plausíveis.

Não é claro, contudo, se linguagem e capacidade de lidar com número são habilidades que evoluíram de maneira interligada (CHOMSKY, 1998; HURFORD, 1987). O domínio da visão, responsável pela decomposição de elementos e cenas complexas por sua vez, também parece atuar de forma recursiva (um tipo de recursividade procedural). A música, considerada como um tipo de linguagem especial, possui a característica de ser hierarquicamente organizada (cf. LERDAHL; JACKENDOFF, 1983; PESETSKY; KATZ, submetido). Recentemente, Pesetsky e Katz (submetido) tem defendido a denominada "tese da identidade para a música e a linguagem". Segundo os autores, todas as diferenças formais entre ambos os sistemas derivam das diferenças entre seus blocos de construção fundamentais: sons e significados arbitrariamente pareados no caso da língua natural e classes de tons e classes de combinações de tons, no caso da música (o que, contudo, parece ser difícil de conciliar com a existência seletiva de amusia e afasias, a menos que os déficits se restrinjam às bases de dados correspondentes (léxico e inventário de sons musicais, sobre os quais operações recursivas operam)). A cognição espacial, que envolve habilidades de navegação e localização, é outro dos domínios que têm sido relacionados à recursividade (BARTLET; KAZAKOV, 2004; KAZAKOV; BARTLET, 2005; ARSENIJEVIC, 2008). Uma série de semelhanças entre a cognição espacial e a língua, dentre as quais o tipo de computações presentes em cada domínio - recursiva e categorial - tem sido salientada (ARSENIJEVIC, 2008). Por último, a teoria da mente - caracterizada como a capacidade que permite 
representar os conhecimentos, pensamentos e sentimentos próprios e dos outros indivíduos - parece ser um domínio no qual a recursividade teria um papel crucial. Tem sido argumentado (DE VILLIERS; DE VILLIERS, 2000) que o desenvolvimento da Teoria da Mente depende crucialmente do suporte de estruturas recursivas (sentenças completivas de verbos de estado mental expressando uma crença falsa em particular). Essa visão pode ser, no entanto, relativizada (CORRÊA et al., a sair; VILLARINHO, 2014).

Retornando à cognição numérica, foco do presente trabalho, além da propriedade da infinitude discreta compartilhada pelos sistemas linguístico e numérico, estruturas recursivas podem ser encontradas em ambos os domínios. Essa ideia é aprofundada a seguir.

\section{Estruturas recursivas na língua e na aritmética}

A computação de expressões numéricas e sentenças da língua parece envolver certos recursos compartilhados; em particular, têm sido apontadas evidências no que diz respeito ao uso, em ambos os casos, da memória verbal de trabalho. Fedorenko, Gibson e Rohde (2007) investigaram a especificidade de domínio dos recursos de memória verbal de trabalho utilizados na integração linguística. Para isso, foram conduzidos experimentos baseados no paradigma de tarefa dual nos quais os participantes deviam executar uma tarefa de compreensão linguística juntamente com uma tarefa secundária envolvendo (i) processos de integração na realização de cálculos aritméticos, ou (ii) processos de integração de informação espacial. Durante os experimentos, ambos os tipos de informação a serem processados foram apresentados simultaneamente na tela de um computador. A depender do experimento, os participantes deviam ler sentenças apresentadas segmento por segmento e, ao mesmo tempo: (i) executar adições simples, também apresentadas por segmentos na condição de processamento aritmético; (ii) realizar tarefas de rotação ou de integração espacial na condição de processamento espacial (cf. a seguir exemplos de cada uma dessas tarefas). 
Figura 1: Tarefas de rotação e integração espacial utilizadas por Fedorenko, Gibson e Rohde (2007)
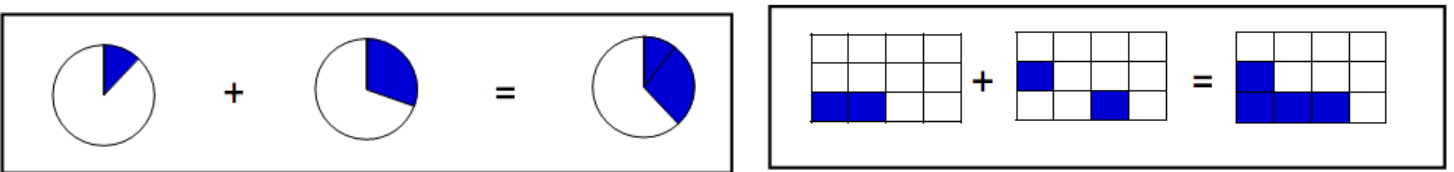

Os resultados obtidos apontaram para uma interação no caso das tarefas envolvendo processamento linguístico e aritmético, mas não no caso do processamento espacial. Diante desses resultados, os autores conjeturam que o processamento linguístico, assim como outras tarefas que requerem da memória verbal de trabalho (no caso, o processamento aritmético), fariam uso dos mesmos recursos.

Outro ponto a partir do qual é possível estabelecer uma analogia diz respeito ao fato de existirem estruturas similares presentes nesses dois domínios. Em termos estritamente estruturais, um paralelo pode ser traçado entre determinadas sentenças e expressões numéricas na medida em que, em ambos os casos, podem ocorrer estruturas envolvendo center-embedding. Com base nessa semelhança estrutural, independentemente do conteúdo de domínio específico, a oração relativa em (8) e a expressão em (9) apresentam uma estrutura recursiva que pode ser representada de forma equivalente, como esquematizado em (10) e (11) respectivamente:

(8) A baleia que a bióloga fotografou mergulhou

(9) $(6-2 \times 2) \times 4$
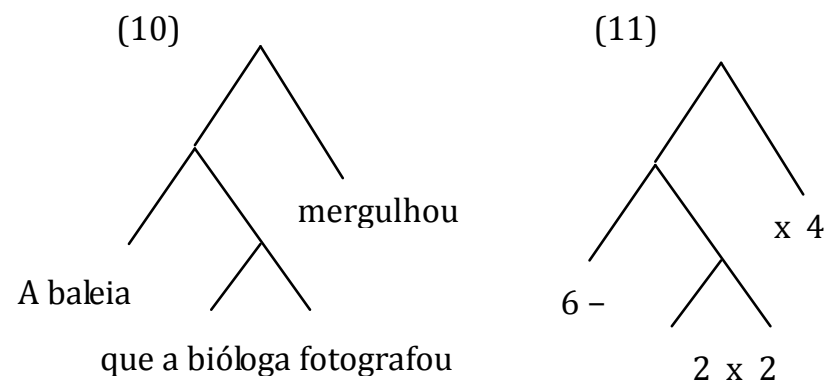

Ambas as estruturas têm em comum o fato de que a formação das relações hierárquicas entre os elementos não é livre, mas obedece a regras específicas. Essas regras 
não necessariamente seguem os mesmos princípios em cada caso, pelas óbvias diferenças entre os domínios, mas mutatis mutandis, o paralelismo em termos estruturais se mantém. Assim sendo, é possível salientar dois aspectos fundamentais que aproximam ambos os tipos de expressões (linguísticas e numéricas): (i) o fato de haver uma distribuição hierárquica que determina a necessidade de manter um elemento na memória até que outras informações sejam computadas; (ii) o fato de, num segundo momento, haver a necessidade de integrar as diferentes informações previamente computadas. No caso específico das expressões numéricas aqui consideradas, as operações de multiplicação/divisão tem prioridade sobre a adição/subtração e, dessa forma, podemos estabelecer um paralelismo com as orações relativas, no sentido de que - em ambos os casos - temos estruturas com center-embedding.

Levando em consideração esses aspectos, haveria alguma relação, em termos de processamento, entre estruturas equivalentes, ainda que associadas a domínios cognitivos diferentes? Nesse caso, a possibilidade de o processamento de um desses tipos de estruturas afetar, em algum grau, o processamento do outro pode ser considerada. 0 fato de um procedimento recursivo ser utilizado em um dado domínio poderia induzir ou facilitar o uso de um procedimento semelhante em outro domínio?

\section{Recursividade na língua e no processamento de expressões numéricas: evidências experimentais}

Nos âmbitos da Psicologia Cognitiva e da Psicolinguística, o fato de o processamento de um determinado item afetar em algum grau o processamento subsequente tem sido explicado com base na noção de priming e a possibilidade de um efeito desse tipo ser obtido vem sendo explorada por meio de técnicas que gerem um efeito de priming. Tal efeito está associado à memória implícita e diz respeito à influência que um evento antecedente (prime) tem sobre o desempenho de um evento posterior (alvo). Esse efeito pode ser tanto de tipo positivo (isto é, quando as pessoas apresentam uma tendência a responder mais depressa a um item precedido de outro item semelhante) ou negativo (quando o prime faz com que o tempo de reação frente ao alvo seja mais lento, gerando assim um efeito 
inibidor).

Embora os efeitos de priming semântico e fonológico sejam os mais explorados, há também um bom número de estudos que visa a captar experimentalmente o efeito da chamada persistência sintática ou priming sintático/estrutural, comumente observado na língua em uso (cf. Pickering e Branigan, 1999 para uma revisão). O priming sintático pode ser caracterizado como a facilitação do processamento que ocorre quando uma dada sentença apresenta a mesma forma sintática que a sentença precedente, o que sugere que a representação decorrente do processamento sintático de uma dada oração pode ser mantida por algum tempo na memória de trabalho, interferindo no processamento sintático subsequente.

Resultados experimentais sugerem que existe uma tendência à repetição de estruturas sintáticas em situações de diálogo (SCHENKEIN, 1980; LEVELT; KELTER, 1982; ESTIVAL, 1985; TANNEN, 1989), na produção de sentenças isoladas em contexto experimental (BOCK, 1986; BOCK; LOEBELL, 1990; POTTER; LOMBARDI, 1998) e também na escrita, embora o efeito de priming pareça ser menos duradouro nessa última modalidade (BRANIGAN; PICKERING, 1999). Bock (1986) mostrou que o tipo de estrutura utilizada na descrição verbal de imagens é afetado pela estrutura das sentenças previamente apresentadas. Estudos recentes têm apontado ainda para efeitos de priming estrutural na compreensão (LEDOUX et al., 2007). Assim sendo, ainda que diferentes áreas cerebrais pareçam estar envolvidas na condução das operações vinculadas a diferentes domínios cognitivos (FRIEDERICH; FRIEDERICI, 2009), a possibilidade de um efeito de priming entre domínios não pode ser, em princípio, descartada. Diante disso, um experimento foi concebido com o intuito de explorar um possível efeito de facilitação no processamento de estruturas envolvendo recursividade independentemente do domínio específico. Para tais fins, foi concebida uma tarefa experimental inspirada na técnica da leitura auto-monitorada (self-paced reading) e na noção de priming sintático.

O paradigma da leitura auto-monitorada permite que o próprio participante controle o tempo de exposição de cada item (palavra, segmento ou frase inteira) durante sua leitura. Assume-se que o tempo levado para apertar o botão que controla a apresentação dos estímulos depende das propriedades daquilo que está sendo lido e se relaciona com o curso 
dos processos cognitivos durante a leitura e a compreensão. A variável dependente considerada é relativa aos tempos de leitura (medida on-line): de cada segmento, da frase completa, de algum dos segmentos previamente definido. No experimento que reportamos a seguir, as sentenças experimentais (orações relativas ou expressões matemáticas) foram apresentadas de forma completa na tela. Os participantes deviam ler o mais rápido possível uma sentença na tela do computador e, posteriormente, responder a uma tarefa de compreensão ${ }^{2}$ que consistia em determinar se a nova informação apresentada era ou não compatível com aquilo que tinha sido lido inicialmente.

O objetivo do experimento conduzido foi avaliar em que medida o processamento de uma estrutura com encaixamento no centro, vinculada a um determinado domínio cognitivo (como a linguagem), influencia o processamento de uma estrutura similar, mas associada a um domínio diferente (como a cognição numérica, ou vice-versa). Considera-se a possibilidade de, uma vez que o participante reconheça uma estrutura recursiva no domínio da linguagem ou no domínio da cognição numérica, o acionamento de um procedimento recursivo venha influenciar o acionamento de um procedimento também recursivo em outro domínio. Trata-se, portanto, de um priming não da estrutura em si, mas do procedimento acionado que gera uma estrutura equivalente.

O tipo de expressão (orações relativas/expressões numéricas) foi considerado como variável independente. Os trials foram separados em dois blocos (orações relativas e expressões numéricas), com vistas a verificar a direção de um possível efeito - seja de facilitação ou de inibição - sendo a ordem de apresentação (leitura de relativas/expressões ou leitura de expressões/relativas) dos mesmos tomada como variável grupal. As variáveis dependentes consideradas foram o tempo de leitura de cada tipo de expressão e o número de respostas-alvo na tarefa de compreensão.

\footnotetext{
2 Experimentos de leitura automonitorada costumam apresentar uma pergunta de compreensão após cada frase com o intuito de verificar o nível de atenção do participante. No experimento aqui reportado, após a leitura, o participante devia decidir se uma nova informação que aparecia na tela (uma nova oração/uma equivalência numérica) combinava ou não com a frase previamente apresentada. Por esse motivo, chamamos essa etapa de "tarefa de compreensão", já que não era feita uma pergunta direta aos participantes.
} 


\subsection{Metodologia}

Participantes

37 adultos, estudantes universitários falantes nativos de português brasileiro, foram avaliados, dos quais foram excluídos três porque não seguiram a instrução de realizar a tarefa o mais rápido possível ou porque apresentaram um número muito elevado de erros na interpretação das expressões numéricas. Esse último fato foi tomado como indicativo de que não dominavam as regras a serem aplicadas. Assim sendo, na análise dos resultados foram considerados os dados correspondentes a 34 participantes (17 em cada grupo. Idade média 29 anos/ intervalo 18-61 anos de idade 3 , 10 homens e 23 mulheres).

\section{Materiais}

Foram utilizados dois blocos com 20 sentenças experimentais em cada condição (orações relativas/ expressões numéricas). A seguir oferecemos alguns exemplos:

(12) Oração relativa: 0 jogador que a torcedora abraçou escorregou.

Tarefa de compreensão: A torcedora abraçou o jogador.

Expressão: $(8-2 \times 2)-1$

Tarefa de compreensão: $=3$

O número de respostas SIM/NÃO foi contrabalançado (metade dos trials em cada condição para cada tipo de resposta correta). Nas orações relativas, a informação apresentada no segundo momento coincidia com a frase inicial ao combinar $\mathrm{N}+\mathrm{V}$ da matriz (13) ou $\mathrm{N}+\mathrm{V}$ da relativa (14):

(13) O menino que a médica examinou emagreceu - 0 menino emagreceu (informação SIM combina).

(14) A tartaruga que a pesquisadora protegeu sumiu - A pesquisadora protegeu a tartaruga (informação SIM combina).

\footnotetext{
${ }^{3} 0$ desempenho de participantes com idade acima dos 50 anos não diferiu do de participantes jovens.
} 
Nas expressões numéricas os trials cuja resposta na tarefa de compreensão era SIM apresentavam a equivalência correta (15):

(15) $(5+2 \times 3) \times 2=22$ (SIM combina).

No que diz respeito aos estímulos com resposta correta NÃO também foram utilizados dois tipos. Nas orações relativas, $\mathrm{N}$ da matriz $+\mathrm{V}$ da relativa (16) ou $\mathrm{N}$ da relativa + V da matriz (17). Nas expressões, um resultado obtido se o cálculo fosse feito de forma linear (i.e. sem respeitar as regras e a estrutura recursiva) (18) ou um resultado parcial obtido se algum passo fosse omitido (19).

(16) A celebridade que o fotógrafo perseguiu fugiu - A celebridade perseguiu o fotógrafo (NÃo combina)

(17) O passageiro que a aeromoça serviu acordou - A aeromoça acordou (NÃo combina)

(18) $(2+3 \times 2) \times 2=20$ (NÃO combina)

(19) $(7-6: 3) \times 4=5$ (NÃO combina)

Aparato

Para a apresentação do material foi utilizado um laptop Apple MacBook A1181, e o experimento foi programado e implementado utilizando o software PsyScope X B53, que permite projetar e monitorar experimentos psicolinguísticos, registrando as respostas dos participantes e o tempo de reação em milésimos de segundos.

\section{Procedimento}

A tarefa utilizada foi concebida, como já mencionado, com base na técnica da leitura auto-monitorada e na noção de priming. 0 participante devia ler as frases que apareciam na tela do computador (orações relativas/expressões numéricas), sendo o próprio sujeito quem controlava a entrada e saída das frases na tela, apertando a barra de espaços num teclado. Após cada frase, era apresentada uma tarefa de compreensão que consistia em decidir se a nova informação recebida combinava ou não com a anterior. A resposta 
(SIM/NÃO) era fornecida também através do teclado. Os estímulos apareciam em ordem aleatória, controlada pelo programa PsyScope, em cada um dos blocos experimentais. Os participantes eram instruídos para realizar o procedimento o mais rápido possível. Cada sessão experimental durou entre 10 e 13 minutos. Segue abaixo um exemplo esquematizado do procedimento.

Figura 2: Exemplo do procedimento

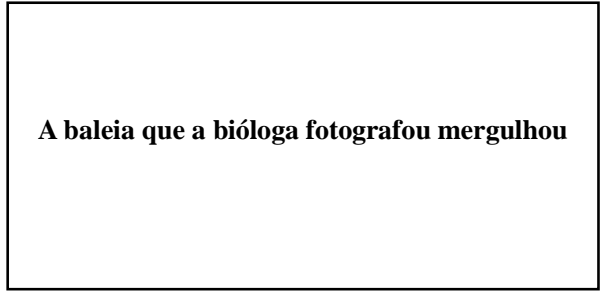

Tela 1

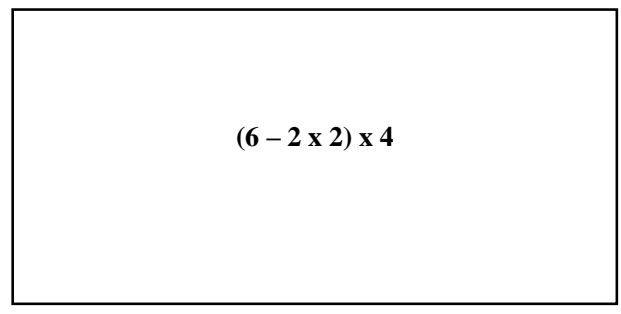

Tela 1

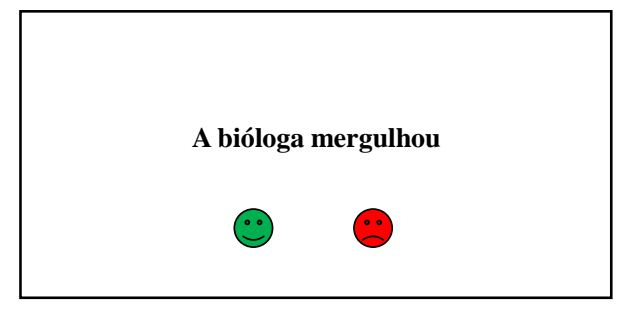

Tela 2

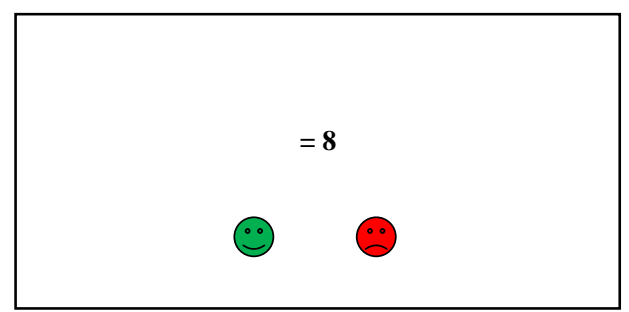

Tela 2

\subsection{Resultados}

Os dados obtidos foram submetidos à análise estatística. Um teste ANOVA com design fatorial 2X2 (ordem de apresentação X tipo de expressão) revelou um efeito principal de tipo de expressão $(\mathrm{F}(1,32)=59.5 \mathrm{p}<.000001)$ com um menor tempo de leitura para as orações relativas do que para as expressões numéricas. 0 Gráfico 1 a seguir ilustra esse efeito. 
Gráfico 1: Tempo médio de leitura em função de tipo de expressão

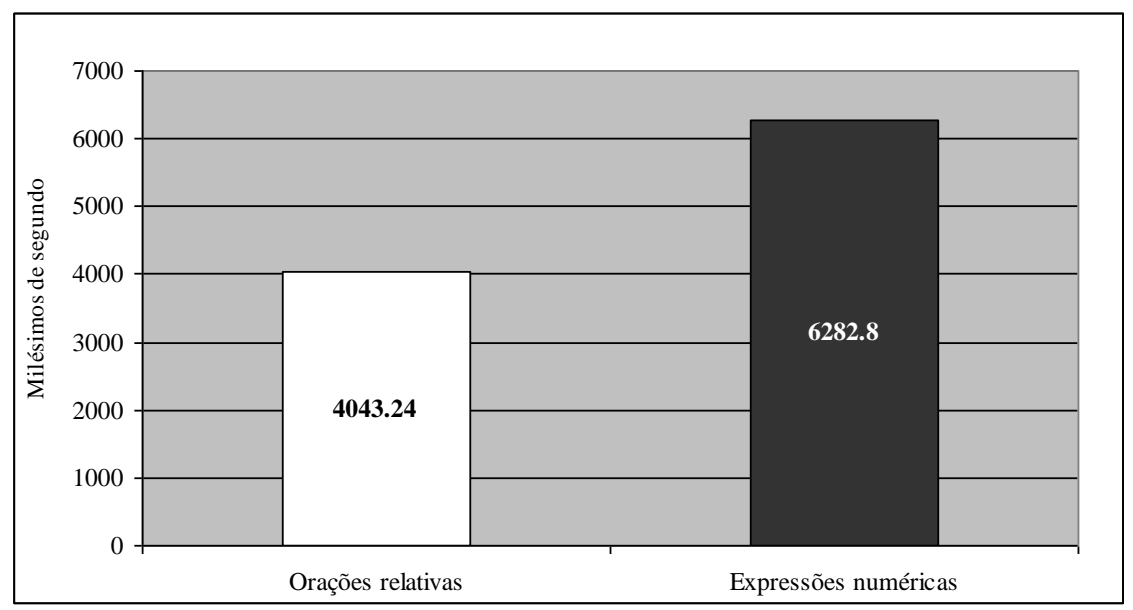

Não foi registrado efeito principal de ordem de apresentação $(\mathrm{F}(1,32)=0.001 \mathrm{p}=.97)$ nem da interação entre as duas variáveis consideradas $(F(1,32)=0.153 \mathrm{p}=.7)$. Independentemente de ordem de apresentação, foram registrados tempos de reação maiores para a leitura das expressões numéricas.

A análise das respostas-alvo na tarefa de compreensão revelou um efeito marginalmente significativo de ordem de apresentação $(\mathrm{t}(16)=2.5 \mathrm{p}=.05)$, com mais respostas-alvo para as orações relativas na ordem de apresentação 2, isto é, quando o primeiro bloco de estímulos apresentado foi o das expressões numéricas (cf. Gráfico 2). No entanto, esse efeito só foi observado no caso das sentenças relativas que tinham como resposta-alvo SIM. Em outras palavras, quando o participante devia responder positivamente na tarefa de compreensão (Ex. O menino que a médica examinou emagreceu - O menino emagreceu), o fato de haver resolvido as expressões numéricas previamente parece de algum modo ter facilitado a compreensão das orações relativas. Alguns dos participantes que realizaram o experimento de acordo com a ordem 2 manifestaram, após o teste, que as orações relativas pareciam "muito fáceis" depois de terem realizado os cálculos. Embora seja um dado anedótico, essa impressão parece ser corroborada pelo resultado obtido na tarefa de compreensão. Esse efeito não foi observado, contudo, quando a resposta esperada era NÃO (Ex. O passageiro que a aeromoça serviu acordou - $A$ aeromoça acordou). 
Gráfico 2: Média de respostas-alvo para as orações relativas na tarefa de compreensão em função de ordem de apresentação e tipo de resposta (SIM/NÃO)

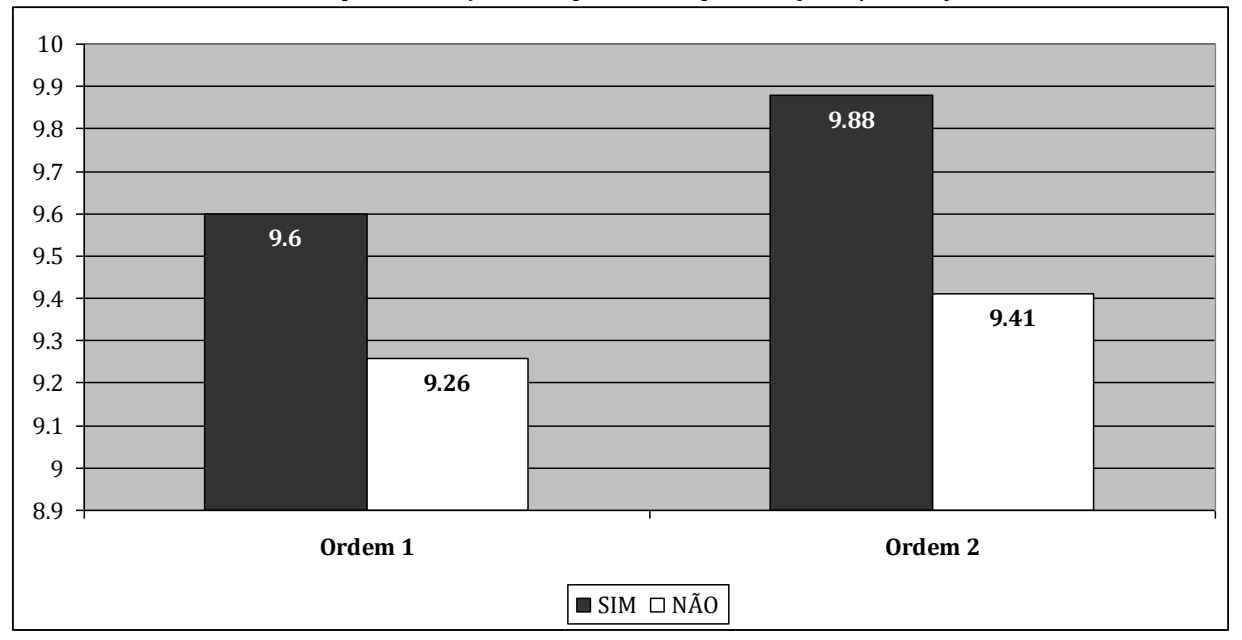

Não foram obtidos outros efeitos quando considerado o número de respostas-alvo na tarefa de compreensão como variável dependente.

\subsection{Discussão}

Os resultados obtidos não sustentam a ideia de que o processamento de estruturas semelhantes - no que diz respeito ao encaixamento recursivo de constituintes - vinculadas a domínios cognitivos diferentes acarrete um efeito de priming estrutural. Assim sendo, apesar de a memória verbal de trabalho (ou loop fonológico, no modelo de Baddeley e Hitch, 1974 e Baddeley, 1986) parecer estar envolvida tanto no processamento de sentenças da língua, quanto de expressões numéricas, sugerindo certo grau de compartilhamento de recursos entre ambos os domínios (FEDORENKO; GIBSON; ROHDE, 2007), tal compartilhamento não sustenta a possibilidade de um efeito de priming a partir do acionamento de um procedimento recursivo no processamento de sentenças e expressões numéricas.

Nossos resultados sugerem que o fato de a informação que desencadeia o uso de procedimentos recursivos de análise (parsing no caso da língua; cálculo, no caso de expressões matemáticas) ser específica de domínio (o pronome relativo, um elemento funcional no caso de expressões linguísticas; sinais de operações e símbolos que indicam os 
passos sequenciais do algoritmo de cálculo, no caso de expressões numéricas) impede que o desencadeamento de um processo em um domínio afete o desencadeamento de processo semelhante em outro domínio, ainda que ambos possam fazer uso de um sistema de memória compartilhado.

A partir desses resultados, consideraremos um possível modo como o uso de um procedimento recursivo seria desencadeado no parsing de sentenças linguísticas e no processo de análise de expressões numéricas, levanto em conta suas especificidades. Partindo-se de um modelo de computação on-line que incorpora uma concepção minimalista de língua (CORRÊA; AUGUSTO, 2007; 2011), toda a informação relevante para o parsing e para a interpretação semântica de sentenças linguísticas encontra-se acessível nas interfaces da língua com os chamados sistemas de desempenho. Assim sendo, o processador linguístico humano será capaz de reconhecer o pronome relativo que (no caso do português) como informação captável na interface fônica (uma vez que se encontra no inventário das formas fônicas que possibilitam o acesso a elementos funcionais no léxico mental) e interpretável na interface semântica. Uma vez que as propriedades sintáticas deste elemento funcional tenham sido recuperadas do léxico, o NP linearmente anteposto a este é identificado como o head da oração relativa e mantido na memória de trabalho até que seus traços (propriedades sintáticas/semânticas) possam ser reativados na posição do gap na sentença encaixada (ou ramificada) que o pronome relativo introduz (ou seja, na posição do elemento movido por meio de uma operação sintática). Nesse ponto, o papel temático do elemento movido (DP sujeito, objeto ou outro) pode ser atribuído. Isso posto, o desencadeamento de um procedimento recursivo na computação sintática (o processamento de uma oração encaixada em um constituinte de outra, em processamento) mostra-se dependente da identificação de informação especificamente linguística (informação pertinente às propriedades sintáticas ou traços formais) do elemento funcional “pronome relativo” no léxico (CORRÊA et al., 2010; AUGUSTO; CORREAA; FORSTER, 2012).

No caso do desencadeamento de um procedimento recursivo para a resolução de uma expressão matemática, no entanto, o processador tem de recuperar a informação que os símbolos utilizados (sinais de operações aritméticas e parênteses, no caso) codificam. Esses símbolos fazem parte de um inventário específico do domínio dos sistemas formais, 
na matemática. Logo, a especificidade de domínio relativa aos elementos disponíveis nas interfaces entre a língua ou de outro tipo de sistema formal e os sistemas que com estes possam fazer interface (sistemas perceptuais e conceituais/intencionais, no caso da língua, e a partir destes sistemas lógico-dedutivos, dentre outros; sistemas perceptuais e sistemas de cálculos, no caso da aritmética, subordinados a sistemas lógico-dedutivos) parece ser crucial para impedir um efeito de ativação (priming) do procedimento recursivo entre domínios, independentemente do quanto as relações hierárquicas que se estabelecem em cada domínio possam ser semelhantes.

Assim sendo, os presentes resultados negativos são compatíveis com resultados que sugerem haver recursos neuronais distintos para a computação sintática e matemática, tais como os reportados por Friedrich e Friederici (2009). Nessa pesquisa, foi investigado o processamento sintático de fórmulas matemáticas abstratas com base em um experimento de leitura utilizando ressonância magnética funcional (fMRI). Os autores partiram da tese de que linguagens formais e línguas naturais compartilham o fato de envolverem uma gramática subjacente encarregada da geração de expressões hierárquicas estruturadas que permite determinar se uma expressão é gramatical ou não (i.e. sintaticamente bem formada). Com base nessa ideia, era prevista uma ativação no giro frontal inferior esquerdo e, em particular, no complexo de Broca - região normalmente ativada durante o processamento de estruturas sintáticas hierárquicas nas gramáticas artificiais (FRIEDERICI et al., 2006a; OPITZ; FRIEDERICI, 2007) e nas línguas naturais (FRIEDERICI et al., 2006b; BEN-SHACHAR et al., 2003). Os resultados revelaram, contudo, que o processamento de fórmulas matemáticas (hierárquica vs. não hierarquicamente estruturadas) ativa uma região no córtex inferior frontal, qual seja, a porção ventral das regiões 45/47 das áreas de Brodman 1 , que é mais anterior e mais ventral que a área do cérebro à qual tem sido atribuído papel crucial no processamento de relações hierárquicas vs. não-hierárquicas na linguagem natural. Com base nesses resultados, os autores defendem a ideia segundo a qual, a despeito das possíveis analogias levantadas, no nível neural, matemática e língua

\footnotetext{
1 As áreas de Brodmann delimitam regiões na citoarquitetura do córtex cerebral, isto é a organização do córtex de acordo com os tecidos formados por células nervosas. Essas áreas foram determinadas por Korbinian Brodmann (1909) e enumeradas de 1 a 52.
} 
natural são processadas de forma diferenciada.

Não é claro, no entanto, onde essa diferenciação reside, quando se busca relacionar computação on-line e sua implementação em nível neuronal. Resultados obtidos por meio de neuroimagem apresentam-se em um grau de granularidade muito superior ao de modelos linguísticos e psicolinguísticos. Assim sendo, a possibilidade de a especificidade da computação por domínio cognitivo (sugerida em resultados recentes Friedrich e Friederici (2009)) se restringir às bases de dados distintas recuperadas em cada caso (o léxico mental, no caso de expressões linguísticas, e o inventário de símbolos da aritmética, no caso das expressões matemáticas), como aventado em Pesetsky e Katz (submetido), em relação à música, não pode ser desconsiderada.

Em suma, embora uma relação estrutural forte entre línguas naturais e linguagens formais possa ser pressuposta (SCHNEIDER, et al., 2011), é necessário um modelo de computação on-line e a explicitação do modo como procedimentos recursivos são "chamados" a partir de informação perceptualmente identificável na interface com bases de dados específicas, para que possamos buscar relações entre domínios cognitivos e seus correlatos neuronais.

\section{Considerações finais}

Este estudo explorou uma possível relação entre o processamento de expressões linguísticas e expressões numéricas que compartilham propriedades formais. Em primeiro lugar, foi necessário clarificar o que pode ser entendido por recursividade em diferentes contextos para que o uso de um procedimento recursivo entre domínios pudesse ser comparado. Em segundo lugar, foi necessário abstraírem-se as semelhanças formais entre expressões linguísticas e numéricas e caracterizar as demandas que a implementação da computação em ambos os casos impõe à memória de trabalho. Desse modo, foi possível desenhar um experimento com vistas a explorar possíveis relações entre domínios.

Constatou-se que, a despeito de o processamento dessas expressões depender crucialmente da memória de trabalho (o que pode requerer o uso de estratégias de ensaio para a manutenção de um elemento ativo até que possa ser recuperado em processos 
subsequentes), o uso de um procedimento recursivo em um domínio não serve de prime, ou seja, não facilita a ativação de procedimento análogo em outro domínio. Esses resultados chamam atenção para a necessidade de se levar em conta o que pode ser visto como informação de interface entre sistemas perceptuais e domínios cognitivos. No caso da língua, considera-se que um modelo de computação on-line que incorpore esse conceito em sua materialidade fônica/visual, integrado a um modelo de parsing/interpretação, é fundamental, dado que semelhanças entre sistemas formais, considerados apenas abstratamente, podem levar a relações ilusórias, no que diz respeito à condução do processamento.

O conceito de interface na perspectiva da computação on-line também possibilitou a discussão dos resultados aqui obtidos em relação aos resultados e hipóteses da neurociência da linguagem. Ao se buscar explicitar de que modo operações sintáticas podem ser conduzidas a partir da informação disponível nas interfaces pertinentes, fica claro que havendo bases de dados distintas por domínios, não é, em princípio, necessário supor procedimentos computacionais distintos entre domínios. A especificidade da informação codificada em cada base pode, em princípio, dar conta das diferenças constatadas. Muito ainda tem de ser investigado nos termos das possíveis relações entre os níveis computacional, representacional/algorítmico e implementacional a partir de possíveis analogias formais entre domínios.

\section{Referências}

ARNOW, David; WEISS, Gerald. Introduction to programming using Java: An object-oriented approach. Reading, MA: Addison Wesley, 1998.

ARSENIJEVIC, Boban. From spatial cognition to language. Biolinguistics, v. 2, n. 1, p. 03-23, 2008.

ARSENIJEVIC, Boban; HINZEN, Wolfram. Recursion as human universal and as a primitive. Biolinguistics, v. 4, n. 2-3, p. 165-173, 2010. 
AUGUSTO, Marina R. A.; CORREA, Letícia. M. S.; FORSTER, Renê. An argument for DPs as phases in an integrated model of on-line computation: the immediate mapping of complex DPs with relative clauses. Revista Virtual de Estudos da Linguagem, v. 6, p. 7-26, 2012.

BADDELEY, Alan D. Working memory. New York: Oxford University Press, 1986.

BADDELEY, Alan D.; HITCH, Graham. Working memory. In: Gordon H. Bower (Ed.). The psychology of learning and motivation. New York: Academic Press, 1974.

BARTLETT, Mark; KAZAKOV, Dimitar. The origins of syntax: from navigation to language. Connection Science, v. 17, p. 271-288, 2005.

BEN-SHACHAR, Michael et al. The neural reality of syntactic transformations - evidence from fMRI. Psychological Science, v. 14, n. 5, p. 433-440, 2003.

BOCK, Kathryn. Syntactic persistence in language production. Cognitive Psychology, v. 18, p. 355-387, 1986.

BOCK, Kathryn; LOEBELL, Helga. Framing sentences. Cognition, v. 35, p.1-39, 1990.

BRANIGAN, Holly; PICKERING, Martin; CLELAND, Alexandra. Syntactic priming in written production: Evidence for rapid decay. Psychonomic Bulletin \& Review, v. 6, n. 4, p. 635-640, 1999.

ĆAVAR, Damir; ĆAVAR, Malgorzata. Inducing recursion. Recursion in human languages Workshop, 2007.

CHOMSKY, Noam. Three models for the description of language. IRE Transactions of Information Theory, IT-2 v. 3, p. 113-124, 1956.

Aspects of the theory of syntax. Cambridge, MA: MIT Press, 1965.

Language and problems of knowledge. MIT Press, Cambridge, Massachusetts, 1998.

New Horizons in the study of language and mind. Cambridge University Press, 2000.

. On minds and language. Biolinguistics, v. 1, p. 9-27, 2007.

CORBALLIS, Michael. The uniqueness of human recursive thinking. American Scientist, v. 95, p. 240-248, 2007.

CORREA, Letícia M. S.; AUGUSTO, Marina R. A.; MARCILESE, M.; VILLARINHO, C. Recursion in language and the development of higher cognitive functions: an investigation with children acquiring Brazilian Portuguese, a sair.

CORREA, Letícia M. S.; AUGUSTO, Marina R. A. Possible loci of SLI from a both linguistic and psycholinguistic perspective. Lingua, v. 121, p. 476-486, 2011. 
- Computação linguística no processamento on-line: soluções formais para a incorporação de uma derivação minimalista em modelos de processamento. Cadernos de Estudos Linguísticos (UNICAMP), v. 49, p. 167-183, 2007.

CORVER, Norbert; DOETJES, Jenny; ZWARTS, Joost. Linguistics perspectives on numerical expressions: Introduction. Lingua, v. 117, p. 751-757, 2007.

DE VILLIERS, Jill; DE VILLIERS, Peter. In: Linguistic determinism and false belief. MITCHELL, P.; RIGGS, K. (Eds). Children's Reasoning and the Mind. Hove, UK: Psychology Press, 2000.

ESTIVAL, Dominique. Syntactic priming of the passive in English. Text, v. 5, p. 7-21, 1985.

EVERETT, Dan. Cultural constraints on grammar and cognition in Pirahã. Current Antropology, v. 46, p. 621-646, 2005.

. Cultural constraints on grammar in Pirahã: A Reply to Nevins, Pesetky and Rodrigues. http://ling.auf.net/lingbuzz/@KpzUAMZAMrzCftFt/BGWiDueZ?244, 2007.

FEDORENKO, Evelina; GIBSON, Edward; ROHDE, Douglas. The nature of working memory in linguistic, arithmetic and spatial integration processes. Journal of Memory and Language, v. 56, n. 2, 2007.

FERREIRA DA SILVA, Shana. A recursividade e seu papel na faculdade da linguagem da espécie humana. Mestrado em Letras (Dissertação), Universidade Federal de Rio Grande do Sul, 2014.

FITCH, Tecumseh; HAUSER, Mark. Computational constraints on syntactic processing in a nonhuman primate. Science, v. 303, p. 377-380, 2004.

FITCH, Tecumseh; CHOMSKY, Noam; HAUSER, Mark. The evolution of the language faculty: Clarifications and implications. Cognition, v. 97, p. 179-210, 2005.

FRIEDERICI, Angela et al. The brain differentiates human and non-human grammars: Functional localization and structural connectivity. PNAS, v. 108, n.7, p. 2458-2463, 2006 .

FRIEDERICI, Angela et al. Processing linguistic complexity and grammaticality in the left frontal cortex. Cerebral Cortex, v. 16, p. 1709-1717, 2006b.

FRIEDRICH, Roland; FRIEDERICI, Angela. Mathematical logic in the human brain syntax. Plos One, v. 4, n. 4, 2009.

HAUSER, Mark; CHOMSKY, Noam; FITCH, Tecumseh. The faculty of language: What is it, who has it, and how did it evolve? Science, v. 298, p. 1569-1579, 2002.

HAREL, David. Algorithmics: the spirit of computing. Reading, MA: Addison- Wesley, 1993. 
HURFORD, Jim. Language and number: The emergence of a cognitive system. Blackwell, Oxford, UK, 1987.

KARLSSON, Fred. Syntactic Recursion and Iteration. In HULST, H. Recursion in Human Language. Berlin/New York: Mouton de Gruyter, 2010.

KAZAKOV, Dimitar; BARTLETT, Mark. Co-operative navigation and the faculty of language. Applied Artificial Intelligence, v. 18, p. 885-901, 2004.

LEDOUX, Kerry; TRAXLER, Matthew; SWAAB, Tamara. Y. Syntactic priming in comprehension: Evidence from Event-Related Potentials. Psychology Science, v. 18, n. 2, p. 135-143, 2007.

LERDAHL, Fred; JACKENDOFF, Ray. A Generative Theory of Tonal Music. Cambridge, Mass.: MIT Press, 1983.

LEVELT, Willem; KELTER, Stephanie. Surface form and memory in question answering. Cognitive Psychology, v. 14, p. 78-106, 1982.

LIGHTFOOT, David W. Introduction. In: CHOMSKY, Noam. Syntactic Structures. Mouton de Gruyter, Berlin, 2002 [1957].

LOBECK, Anne. Discovering grammar: An introduction to English sentence structure. New York: Oxford University Press, 2000.

LOUDON, Kyle. Mastering algorithms with C. Cambridge, MA: O'Reilly, 1999.

LOBINA, David; GARCÍA-ALBEA, José Eugenio. Recursion and cognitive science: Data structure and mechanisms. Proceedings of the 31th Annual Conference of the Cognitive Science Society, 2009.

MARCILESE, Mercedes. Sobre o papel da língua no desenvolvimento de habilidades cognitivas superiores: representação, recursividade e cognição numérica. Tese de Doutorado, Pontifícia Universidade Católica do Rio de Janeiro, 2011.

NEVINS, Andrew; PESETSKY, David; RODRIGUES, Cilene. Pirahã's Excepcionality: a Reassessment, 2007.

OPITZ, Bertram; FRIEDERICI, Angela. Neural bases of processing sequential and hierarquical syntactic structures. Human Brain Mapping, v. 28, p. 585-592, 2007.

PARISI, Domenico. Introduzione a la psicolinguistica. Firenze: Le Monnier, 1981.

PARKER, Anna. Evolution as a constraint on theories of syntax: the case against Minimalism. $\mathrm{PhD}$ Thesis, University of Edinburgh, 2006a. 
. Evolving the narrow language faculty: was recursion the pivotal step? Language Evolution and Computation Research Unit, University of Edinburgh, Scotland, 2006b.

PESETSKY, David; KATZ, Jonah. The identity thesis for language and music, submetido. Disponível em: lingBuzz/000959.

PICKERING, Martin; BRANIGAN, Holly. Syntactic priming in language production. Trends in Cognitive Sciences, v. 3, n. 4, p. 136-141, 1999.

PINKER, Steven; JACKENDOFF, Ray. The Faculty of language: what's special about it? Cognition, v. 95, p. 201-236, 2005.

POTTER, Mary; LOMBARDI, Linda. Syntactic priming in immediate recall of sentences. Journal of Memory and Language, v. 38, p. 265-282, 1998.

SCHENKEIN, Jim. A taxonomy for repeating action sequences in natural conversation. BUTTERWORTH, Brian (Ed.). Language production. London: Academic Press, 1980.

SCHNEIDER, Elisa; MURAYAMA, Masaka; DEHAENE, Stanislas; SIGMAN, Mariano. Organización sintáctica del pensamiento matemático. Trabalho apresentado no Primer Encuentro de Grupos de Investigación sobre Procesamiento del Lenguaje, Buenos Aires, 2011.

SOARE. Robert. Computability and recursion. The Bulletin of Symbolic Logic, v. 2, n. 3, p. 284321, 1996.

TANNEN, Deborah. Talking voices: Repetition, dialogue and imagery in conversational discourse. Cambridge: Cambridge University Press, 1989.

TOMALIN, Marcus. Reconsidering recursion in syntactic theory. Lingua, v. 117, p. 17841800, 2007.

VILLARINHO, Clara. N. G. Um papel para a língua no desenvolvimento de habilidades cognitivas superiores: o traço de ponto de vista em estruturas completivas e o domínio de crenças falsas. 2012. 205 f. Tese (Doutorado em Estudos da Linguagem) - Programa de PósGraduação em Estudos da Linguagem, Pontifícia Universidade Católica do Rio de Janeiro, Rio de Janeiro, 2012.

VINOGRADOV, Ivan Matveevich. (Ed.). Encyclopaedia of Mathematics. Volume 8. Netherlands: Kluwer Academic Publishers, 1992.

Recebido em março de 2014.

Aceito em maio de 2014. 